\title{
Corrigendum: A Student's Guide to Neural Circuit Tracing
}

\author{
Christine Saleeba ${ }^{1,2+}$, Bowen Dempsey ${ }^{3 \dagger}$, Sheng Le ${ }^{1}$, Ann Goodchild ${ }^{1}$ and \\ Simon McMullan ${ }^{1 *}$ \\ ${ }^{1}$ Neurobiology of Vital Systems Node, Faculty of Medicine and Health Sciences, Macquarie University, Sydney, NSW, \\ Australia, ${ }^{2}$ The School of Physiology, Pharmacology and Neuroscience, University of Bristol, Bristol, United Kingdom, \\ ${ }^{3}$ CNRS, Hindbrain Integrative Neurobiology Laboratory, Neuroscience Paris-Saclay Institute (Neuro-PSI), Université \\ Paris-Saclay, Gif-sur-Yvette, France
}

Keywords: neuroanatomy, viral tracers, anterograde tracer, retrograde tracers, synaptic contacts, connectome analysis

\section{A Corrigendum on}

\section{A Student's Guide to Neural Circuit Tracing} by Saleeba, C., Dempsey, B., Le, S., Goodchild, A., and McMullan, S. (2019). Front. Neurosci. 13:897. doi: 10.3389/fnins.2019.00897

In the original article, there was an error: one section of our review considers reagents traditionally considered to be anterograde tracers (i.e., fluorescent or antigenic substances that are taken up by neuronal cell bodies at the site of application and transported to the synaptic terminals). The original text read:

Emerging in the mid-1980s, dextran-based tracers, particularly biotinylated dextran amine (BDA), were rapidly adopted and remain one of the most widely used conventional anterograde tracers (Glover et al., 1986; Brandt and Apkarian, 1992; Veenman et al., 1992; Wouterlood et al., 2014). BDA enters injured neurons at the injection site, undergoes rapid anterograde transport and spreads evenly throughout the entire neuron, resulting in a Golgi-like level of staining detail (Köbbert et al., 2000; Lanciego and Wouterlood, 2011; Wouterlood et al., 2014). Interestingly, while $10 \mathrm{kDa}$ BDA travels mostly anterogradely, the $3 \mathrm{kDa}$ form is a retrograde tracer (Reiner et al., 2000; Lanciego and Wouterlood, 2011). Like CTb, fluorophore-labeled dextran amine variants are now widely used instead of biotinylated versions that require histological processing for visualization, and a number of authors have used tetramethylrhodamine-conjugated dextran for juxtacellular labeling during electrophysiological recordings (Noseda et al., 2010; Dempsey et al., 2015).

\section{Limitations of Conventional Tracers}

Despite their ongoing popularity, the major limitations of conventional tracers are worthy of consideration:

(1) Conventional tracers are taken up by fibers of passage (Dado et al., 1990; Chen and AstonJones, 1995; Conte et al., 2009), which can lead to incorrect identification of projections. [Notably, canine adenovirus (CAV) can also be taken up by fibers of passage (Schwarz et al., 2015)].

Received: 04 February 2020 Accepted: 17 February 2020

Published: 10 March 2020

Citation:

Saleeba C, Dempsey B, Le S, Goodchild A and McMullan S (2020) Corrigendum: A Student's Guide to Neural Circuit Tracing.

(2) The spread of many conventional tracers around the injection site results in intense and diffuse labeling that may reflect deposition in the extracellular matrix or take-up by neurons or glia. Such non-specific labeling makes it difficult to reliably identify labeled neurons within $\sim 1 \mathrm{~mm}$ of the injection site. Thus the historical use of conventional tracers has probably overemphasized the relative significance of distant inputs/outputs compared to those originating from local interneurons; contemporary connectomic studies indicate that longdistance projections are relatively rare compared to short-distance connections (Oh et al., 2014; Henriksen et al., 2016; van den Heuvel et al., 2016; Dempsey et al., 2017). 
(3) Tracer uptake relies predominantly on sugars that are located on the glycocalyx of most, if not all neurons, or on common mechanisms such as endocytosis. Consequently, restricted uptake by functionally or neurochemically/genetically homogeneous neuronal populations is not possible.

(4) The direction of axonal transport is often not exclusive, which complicates circuit analysis; for example, $\mathrm{CTb}$, perhaps the mostly widely used "retrograde" tracer, is also an efficient anterograde tracer (Luppi et al., 1987; Angelucci et al., 1996; Noseda et al., 2010).

The authors were contacted by Professor Joel Glover, who first described the use of dextran amines as neuronal tracers in the 1980s and expressed concern that we had inadvertently perpetuated a myth regarding the directional sensitivity of these tracers.

The amendment to the article clarifies the bidirectional nature of dextran amine transport.

A correction has been made to the Anterograde and Retrograde Tracers section, subsection Conventional (Mainly) Anterograde Tracers;

"Emerging in the mid-1980s, dextran-amines (DAs) were rapidly adopted and remain widely used as conventional axonal tracers (Gimlich and Braun, 1985; Glover et al., 1986; Brandt and Apkarian, 1992; Veenman et al., 1992; Wouterlood et al., 2014). DAs enter injured neurons at the injection site and spread evenly throughout the entire neuron via diffusion, resulting in a Golgi-like level of staining detail (Glover et al., 1986; Fritzsch, 1993; Glover, 1995; Köbbert et al., 2000; Lanciego and Wouterlood, 2011; Wouterlood et al., 2014).

Despite the common perception that DAs are preferential anterograde tracers, many studies indicate bidirectional travel (Schmued et al., 1990; Fritzsch, 1993; Glover, 1995; Zhang et al., 2017), including the original description of their axonal transport by Glover et al. (1986). Their retrograde capabilities have been exploited both for conventional tracing (Sivertsen et al., 2014, 2016; Lunde et al., 2019) and for delivery of calcium-sensitive indicators for optical recording of neurons selected by axonal trajectory (O'Donovan et al., 1993; McPherson et al., 1997).

There is a perception that the molecular weight of DAconjugates contributes to their directional selectivity, with smaller molecules exhibiting superior performance as a retrograde tracer (Reiner et al., 2000; Lanciego and Wouterlood, 2011). However, the influence, if any, of molecular weight on directional specificity is probably overstated, and may instead reflect differences in speed of transport, which is distinctly

\section{REFERENCES}

Angelucci, A., Clascá, F., and Sur, M. (1996). Anterograde axonal tracing with the subunit B of cholera toxin: a highly sensitive immunohistochemical protocol for revealing fine axonal morphology in adult and neonatal brains. J. Neurosci. Methods 65, 101-112. doi: 10.1016/0165-0270(95)00155-7

Brandt, H. M., and Apkarian, A. V. (1992). Biotin-dextran: a sensitive anterograde tracer for neuroanatomic studies in rat and monkey. J. Neurosci. Methods 45, 35-40. doi: 10.1016/0165-0270(92)90041-b faster for smaller compounds (Fritzsch, 1993), combined with differences in volume of synaptic terminals compared to cell bodies (Joel C Glover, personal communication).

Like CTb, fluorophore-labeled dextran amine variants are now widely used instead of or in addition to biotinylated versions that require histological processing for visualization, and we and others have used tetramethylrhodamine-conjugated dextran for juxtacellular labeling during electrophysiological recordings (Noseda et al., 2010; Dempsey et al., 2015).

\section{Limitations of Conventional Tracers}

Despite their ongoing popularity, the major limitations of conventional tracers are worthy of consideration:

1. Conventional tracers can be taken up by fibers of passage (Dado et al., 1990; Chen and Aston-Jones, 1995; Conte et al., 2009), which can lead to incorrect identification of projections. [Notably, canine adenovirus (CAV) can also be taken up by fibers of passage (Schwarz et al., 2015)].

2. The spread of many conventional tracers around the injection site results in intense and diffuse labeling that may reflect deposition in the extracellular matrix or takeup by neurons or glia. Such non-specific labeling makes it difficult to reliably identify labeled neurons within $\sim 1 \mathrm{~mm}$ of the injection site. Thus the historical use of conventional tracers has probably overemphasized the relative significance of distant inputs/outputs compared to those originating from local interneurons; contemporary connectomic studies indicate that long-distance projections are relatively rare compared to short-distance connections (Oh et al., 2014; Henriksen et al., 2016; van den Heuvel et al., 2016; Dempsey et al., 2017).

3. Tracer uptake relies predominantly on sugars that are located on the glycocalyx of most, if not all neurons, or on common mechanisms such as endocytosis. Consequently, restricted uptake by functionally or neurochemically/genetically homogeneous neuronal populations is not possible.

4. The direction of axonal transport is rarely exclusive, which complicates circuit analysis; the archetypal retrograde and anterograde tracers, $\mathrm{CTb}$ and BDA respectively, both label axons traveling in the "wrong" direction (Luppi et al., 1987; Schmued et al., 1990; Fritzsch, 1993; Glover, 1995; Angelucci et al., 1996; Noseda et al., 2010; Zhang et al., 2017).”

The authors apologize for this error and state that this does not change the scientific conclusions of the article in any way. The original article has been updated. 
Dempsey, B., Le, S., Turner, A., Bokiniec, P., Ramadas, R., Bjaalie, J. G., et al. (2017). Mapping and analysis of the connectome of sympathetic premotor neurons in the rostral ventrolateral medulla of the rat using a volumetric brain atlas. Front. Neural Circ. 11:9. doi: 10.3389/fncir.2017.00009

Dempsey, B., Turner, A. J., Le, S., Sun, Q. J., Bou Farah, L., Allen, A. M., et al. (2015). Recording, labeling, and transfection of single neurons in deep brain structures. Physiol. Rep. 3:e12246. doi: 10.14814/phy2.12246

Fritzsch, B. (1993). Fast axonal diffusion of 3000 molecular weight dextran amines. J. Neurosci. Methods 50, 95-103.

Gimlich, R. L., and Braun, J. (1985). Improved fluorescent compounds for tracing cell lineage. Dev. Biol. 109, 509-514.

Glover, J. C. (1995). Retrograde and anterograde axonal tracing with fluorescent dextran-amines in the embryonic nervous system. Neurosci. Protoc. 95, 1-13.

Glover, J. C., Petursdottir, G., and Jansen, J. K. S. (1986). Fluorescent dextran amines used as axonal tracers in the nervous system of chicken embryo. J. Neurosci. Methods 18, 243-254. doi: 10.1016/0165-0270(86)90011-7

Henriksen, S., Pang, R., and Wronkiewicz, M. (2016). A simple generative model of the mouse mesoscale connectome. eLife 5:e12366. doi: 10.7554/eLife.12366

Köbbert, C., Apps, R., Bechmann, I., Lanciego, J. L., Mey, J., and Thanos, S. (2000). Current concepts in neuroanatomical tracing. Prog. Neurobiol. 62, 327-351. doi: 10.1016/s0301-0082(00)00019-8

Lanciego, J. L., and Wouterlood, F. G. (2011). A half century of experimental neuroanatomical tracing. J. Chem. Neuroanat. 42, 157-183. doi: 10.1016/j.jchemneu.2011.07.001

Lunde, A., Okaty, B. W., Dymecki, S. M., and Glover, J. C. (2019). Molecular profiling defines evolutionarily conserved transcription factor signatures of major vestibulospinal neuron groups. eNeuro 6:e0475-0418.2019. doi: 10.1523/ENEURO.0475-18.2019

Luppi, P. H., Sakai, K., Salvert, D., Fort, P., and Jouvet, M. (1987). Peptidergic hypothalamic afferents to the cat nucleus raphe pallidus as revealed by a double immunostaining technique using unconjugated cholera toxin as a retrograde tracer. Brain Res. 402, 339-345. doi: 10.1016/0006-8993(87)90041-2

McPherson, D. R., McClellan, A. D., and O’Donovan, M. J. (1997). Optical imaging of neuronal activity in tissue labeled by retrograde transport of Calcium Green Dextran. Brain Res. Brain Res. Protoc. 1, 157-164. doi: 10.1016/S1385-299X(96)00024-4

Noseda, R., Kainz, V., Jakubowski, M., Gooley, J. J., Saper, C. B., Digre, K., et al. (2010). A neural mechanism for exacerbation of headache by light. Nat. Neurosci. 13, 239-245. doi: 10.1038/nn.2475

O’Donovan, M. J., Ho, S., Sholomenko, G., and Yee, W. (1993). Real-time imaging of neurons retrogradely and anterogradely labelled with calciumsensitive dyes. J. Neurosci. Methods 46, 91-106. doi: 10.1016/0165-0270(93) 90145-H
Oh, S. W., Harris, J. A., Ng, L., Winslow, B., Cain, N., Mihalas, S., et al. (2014). A mesoscale connectome of the mouse brain. Nature 508, 207-214. doi: $10.1038 /$ nature 13186

Reiner, A., Veenman, C. L., Medina, L., Jiao, Y., Del Mar, N., and Honig, M. G. (2000). Pathway tracing using biotinylated dextran amines. J. Neurosci. Methods 103, 23-37. doi: 10.1016/s0165-0270(00)0 0293-4

Schmued, L., Kyriakidis, K., and Heimer, L. (1990). In vivo anterograde and retrograde axonal transport of the fluorescent rhodamine-dextranamine, Fluoro-Ruby, within the CNS. Brain Res. 526, 127-134. doi: 10.1016/0006-8993(90)90258-D

Schwarz, L. A., Miyamichi, K., Gao, X. J., Beier, K. T., Weissbourd, B., DeLoach, K. E., et al. (2015). Viral-genetic tracing of the input-output organization of a central noradrenaline circuit. Nature 524, 88-92. doi: 10.1038/nature 14600

Sivertsen, M. S., Glover, J. C., and Perreault, M. C. (2014). Organization of pontine reticulospinal inputs to motoneurons controlling axial and limb muscles in the neonatal mouse. J. Neurophysiol. 112, 1628-1643. doi: 10.1152/jn.00820.2013

Sivertsen, M. S., Perreault, M. C., and Glover, J. C. (2016). Pontine reticulospinal projections in the neonatal mouse: Internal organization and axon trajectories. J. Comp. Neurol. 524, 1270-1291. doi: 10.1002/cn e.23904

van den Heuvel, M. P., Bullmore, E. T., and Sporns, O. (2016). Comparative connectomics. Trends Cogn. Sci. 20, 345-361. doi: 10.1016/j.tics.201 6.03.001

Veenman, C. L., Reiner, A., and Honig, M. G. (1992). Biotinylated dextran amine as an anterograde tracer for single- and double-labeling studies. J. Neurosci. Methods 41, 239-254. doi: 10.1016/0165-0270(92)90089-v

Wouterlood, F. G., Bloem, B., Mansvelder, H. D., Luchicchi, A., and Deisseroth, K. (2014). A fourth generation of neuroanatomical tracing techniques: exploiting the offspring of genetic engineering. J. Neurosci. Methods 235, 331-348. doi: 10.1016/j.jneumeth.2014.07.021

Zhang, W. J., Xu, D. S., Cui, J. J., Jing, X. H., Xu, N. G., Liu, J. H., et al. (2017). Anterograde and retrograde tracing with high molecular weight biotinylated dextran amine through thalamocortical and corticothalamic pathways. Microsc. Res. Tech. 80, 260-266. doi: 10.1002/jemt.22797

Copyright (c) 2020 Saleeba, Dempsey, Le, Goodchild and McMullan. This is an openaccess article distributed under the terms of the Creative Commons Attribution License (CC BY). The use, distribution or reproduction in other forums is permitted, provided the original author(s) and the copyright owner(s) are credited and that the original publication in this journal is cited, in accordance with accepted academic practice. No use, distribution or reproduction is permitted which does not comply with these terms. 